\title{
Intersection of the tocopherol and plastoquinol metabolic pathways at the plastoglobule
}

Anna Maria Zbierzak*, Marion Kanwischer ${ }^{\dagger}$, Christina Wille ${ }^{\dagger}$, Pierre-Alexandre Vidi ${ }^{\ddagger}$, Patrick Giavalisco $^{\dagger}$, Antje Lohmann ${ }^{\dagger}$, Isabel Briesen*, Svetlana Porfirova ${ }^{\dagger}$, Claire Bréhélin», Felix Kessler $^{\ddagger}$ and Peter Dörmann*

*Institute of Molecular Physiology and Biotechnology of Plants, University of Bonn, Karlrobert-Kreiten-Strasse 13, 531115 Bonn, Germany;

${ }^{\dagger}$ Max Planck Institute of Molecular Plant Physiology, Department of Molecular Physiology, Am Mühlenberg 1, 14476 Potsdam, Germany;

${ }^{\ddagger}$ Laboratory of Plant Physiology, Institute of Biology, University of Neuchâtel, rue Emile Argand 11, CP 158, CH-2007 Neuchâtel, Switzerland

\section{Corresponding authors:}

Peter Dörmann

Institute of Molecular Physiology and Biotechnology of Plants

University of Bonn

Karlrobert-Kreiten-Strasse 13

531115 Bonn

Germany

Tel.: +49-228-732830

Fax: +49-228-831696

E-mail: doermann@uni-bonn.de

Felix Kessler

Laboratory of Plant Physiology, Institute of Biology

University of Neuchâtel

Rue Emile-Argand 11, CP 158

CH-2009 Neuchâtel

Switzerland

felix.kessler@unine.ch

Short title: Tocochromanols in Arabidopsis

\section{KEY WORDS}

Arabidopsis, tocochromanol, plastoquinone, plastochromanol, plant, chloroplast

\begin{abstract}
ABBREVIATIONS
DMGGBQ, 2,3-dimethyl-5-geranylgeranyl-1,4-benzquinol; DMPBQ, 2,3-dimethyl-5-phytyl1,4-benzoquinol; HPPD, p-hydroxyphenylpyruvate dioxygenase; MGGBQ, 2-methyl-6geranylgeranyl-1,4-benzoquinol; MPBQ, 2-methyl-6-phytyl-1,4-benzoquinol; MPC-8, 5methyl plastochromanol-8; MSBQ, 2-methyl-6-solanesyl-1,4-benzoquinol; PC-8, plastochromanol-8; PQ-9, plastoquinol-9; VTE1, tocopherol cyclase; VTE2, homogentisate phytyltransferase; VTE3, MPBQ, 2-methyl-6-phytyl-1,4-benzoquinol methyltransferase; VTE4, $\gamma$-tocopherol methyltransferase
\end{abstract}




\begin{abstract}
Plastoglobules, lipid-protein bodies in the stroma of plant chloroplasts, are enriched in nonpolar lipids, in particular prenyl quinols. Here we show that in addition to the thylakoids, plastoglobules also contain a considerable proportion of the plastidial plastoquinol-9 (PQ-9), the redox component of photosystem II, and of the cyclized product of PQ-9, plastochromanol8 (PC-8), a tocochromanol with a structure similar to $\gamma$-tocopherol and $\gamma$-tocotrienol, but with a C40 prenyl side chain. PC-8 formation was abolished in the Arabidopsis thaliana tocopherol cyclase mutant vtel, but accumulated in VTE1 overexpression plants, in agreement with a role of tocopherol cyclase (VTE1) in PC-8 synthesis. VTE1 overexpression resulted in the proliferation of the number of plastoglobules which occurred in the form of clusters in the transgenic lines. Simultaneous overexpression of VTE1 and of the methyltransferase VTE4 resulted in the accumulation of a compound tentatively identified as 5-methyl-PC-8, the methylated form of PC-8. Our data suggest that the existence of a plastoglobular pool of PQ-9, along with the partial conversion of PQ-9 into PC-8, might represent a mechanism for the regulation of the antioxidant content in thylakoids and of the PQ-9 pool that is available for photosynthesis.
\end{abstract}




\section{INTRODUCTION}

Organisms performing oxygenic photosynthesis harbor a unique set of prenyl quinols, i.e. phylloquinol (vitamin K1), plastoquinol-9 (PQ-9) and tocochromanols (tocopherol, tocotrienol; vitamin E), which serve as electron carriers in photosystems I and II (PSI, PSII), and as lipid antioxidant, respectively. Eight forms of tocochromanols are commonly considered to occur in plants, i.e. four forms of tocopherol and tocotrienol each, carrying a phytyl or geranylgeranyl side chain, respectively [1]. The individual forms of tocopherols and tocotrienols are distinguished by the number and positions of methyl substituents on the chromanol ring. Tocopherols are synthesized from homogentisate and phytyl-diphosphate by homogentisate phytyltransferase (HPT1, VTE2) [2, 3] (Fig. 1). After methylation by VTE3, the second ring of 2,3-dimethyl-5-phytyl-1,4-benzoquinol (DMPBQ) is closed by tocopherol cyclase (VTE1) yielding $\gamma$-tocopherol [4-7]. Final methylation by $\gamma$-tocopherol methyltransferase (VTE4) results in formation of $\alpha$-tocopherol [8-11] (Fig. 1). In many species including Arabidopsis thaliana, $\alpha$-tocopherol is the most abundant form in leaves, while $\gamma$ tocopherol is predominant in seeds $[8,9]$. Tocopherol is found in the thylakoid membranes of chloroplasts, where it presumably protects the photosynthetic apparatus against oxidative stress. Another fraction of tocopherol is associated with the plastoglobules which is in agreement with the finding that VTE1 was identified as a plastoglobular protein [12-15].

The synthesis of plasotquinol (PQ-9) depends on the prenylation of homogentisate [16] employing a long hydrocarbon side chain derived from solanesyl-diphosphate (nonaprenyldiphosphate). An A. thaliana gene, HST, presumably inyolved in the transfer of the solanesyl group onto homogentisate, was described $[17,18]$. Methylation by VTE3, a methyltransferase shared by the tocopherol and PQ-9 pathways, results in the formation of PQ-9 [4-6]. Two molecules of PQ-9 are associated with photosystem $I_{2}$ one $\left(\mathrm{Q}_{\mathrm{A}}\right)$ which is firmly bound to the PSII reaction center, and a second one $\left(Q_{B}\right)$ which can be exchanged with the mobile pool of PQ-9 in the thylakoid membranes [19]. Furthermore, PQ-9 was identified in the plastoglobules of chloroplasts, but the pool size of plastoglobular PQ-9 and its function remained unknown [20].

Whittle et al. [21] were the first to describe a derivative of PQ-9 in plants which was designated "plastochromanol-8" (PC-8). PC-8 contains the very same head group as $\gamma-$ tocopherol and $\gamma$-tocotrienol ("plastochromanol-3"), but differs with respect to its prenyl side chain which contains 40 carbon atoms ( 8 isoprene units; Fig. 1). PC-8 was detected in rubber trees and in the seed oils of flax, rape and maize [22-26], and later in the seed oils of Turkish pine, hemp and caper [27-29]. The fact that $\gamma$-tocopherol and PC-8 share the same head group suggested that PC-8 is derived from a cyclization reaction of PQ-9 catalyzed by VTE1 (Fig. 1). In agreement with this hypothesis, VTE1 overexpression in seeds of Brassica napus resulted in a simultaneous increase in tocopherols and in PC-8 [17, 30, 31].

To study the localization and function of PQ-9 and PC-8 in leaves, subfractions of chloroplast membranes were isolated and PQ-9 and PC-8 quantified. From these experiments it became clear that a considerable proportion of these two prenyl quinol lipids is associated with plastoglobules. Measurements of prenyl quinols in different $A$. thaliana mutants and transgenic lines revealed that PQ-9/PC-8 metabolism is closely linked with tocopherol metabolism by sharing a number of enzymatic steps.

\section{EXPERIMENTAL}

\section{Plant material}

A. thaliana wild type (Columbia), transgenic and mutant plants were grown with $16 \mathrm{~h}$ light $\left(120 \mu \mathrm{mol} \mathrm{s}^{-1} \mathrm{~m}^{-2}\right)$ per day. Leaf material for chloroplast isolation and fractionation was obtained from six to eight-week old plants grown under an $8 \mathrm{~h} /$ day light regime. The following 
lines were used: vtel, ethylmethane sulfonate mutant carrying a premature stop codon in the tocopherol cyclase gene [7]; vte2-1, ethylmethane sulfonate mutant with an amino acid exchange in the homogentisate phytyltransferase gene [32]; vte2-3, mutant allele carrying a TDNA insertion (line GABI_867G08; Gabi Kat Collection, Bielefeld, Germany) [33]. The position of the T-DNA insertion in vte2-3 was confirmed by PCR using primers for amplification of genomic DNA (PD294, 5'-TGTTCTGGGCTCTTTTTGTGA; PD256, 5'AAATTGGAGCGCATAAAAAGGCAGTA) and of the sequence flanking the insertion (TDNA left boarder primer, 5'-CCCATTTGGACGTGAATGTAGACAC; PD256). Sequencing of the flanking DNA amplified by PCR confirmed the position of the T-DNA in the 11th exon after $\mathrm{Val}^{319}$.

\section{Overexpression of VTE1 and VTE4 in A. thaliana leaves}

Overexpression of the VTE 1 cDNA under control of the cauliflower mosaic virus $35 \mathrm{~S}$ promoter in A. thaliana was described previously [34]. Two lines (WT-VTE1\#40 and vte1VTE1\#1 in WT and vtel background, respectively) showing strong expression of VTE1 in Northern and Western analysis were selected for further analyses. The full-length VTE4 cDNA was released from clone RAFL07-18-C14 (RIKEN BioResource Center, Tsukuba, Japan) with $E c o$ RI, BamHI and ligated into pBluescriptIISK+ (Stratagene). After digestion with KpnI and $X b a \mathrm{I}$, the VTE4 cDNA was cloned behind the $35 \mathrm{~S}$ promoter of the binary vector pBINAR-Hyg [35]. The VTE4 construct was transferred into the line WT-VTE1\#40 by floral dip [36]. Double transgenic lines selected for hygromycin B resistance were screened by fluorescence HPLC for alterations in tocochromanol composition.

\section{Measurement of tocochromanols and plastoquinol-9}

Tocochromanols were isolated from plant leaves with diethylether and $1 \mathrm{M} \mathrm{KCl} / 0.2 \mathrm{M}$ $\mathrm{H}_{3} \mathrm{PO}_{4}$, or from plant seeds with ethanol $[34,37]$. Tocol $(500 \mathrm{ng})$ was added as internal standard. Tocopherol concentrations were adjusted using absorption coefficients as published [38]. The tocochromanols were dissolved in hexane and quantified by fluorescence HPLC [39]. Briefly, tocochromanols were injected onto a Lichrospher 100 diol, $5 \mu \mathrm{m}, 3 \mathrm{~mm} \times 25 \mathrm{~cm}$ column (Knauer, Berlin) attached to an Agilent 1100 HPLC System. The solvent was nhexane/tertiary butylmethylether $(96: 4)$ at a flow rate of $0.75 \mathrm{~mL} \mathrm{~min}^{-1}$. Fluorescence was recorded with an excitation of $290 \mathrm{~nm}$ and emission of $330 \mathrm{~nm}$.

PC-8 was quantified together with tocopherols by fluorescence HPLC (diol column, see above) using tocol as internal standard [39]. The linearity of PC-8 quantification by HPLC was determined using PC-8 isolated from linseed via HPLC. The response factor of PC-8 relative to tocol was determined after adjusting the concentration of PC-8 (isolated from linseed) using the absorption coefficient[24].

PQ-9 was isolated from frozen leaves by extraction with $1 \mathrm{M} \mathrm{KCl} / 0.2 \mathrm{M} \mathrm{H}_{3} \mathrm{PO}_{4}$, and hexane and subsequently with diethylether. Ubiquinone-4 (500 ng; Sigma) was added as internal standard. The combined organic phases were evaporated with air and the residue dissolved in hexane. PQ-9 was quantified by HPLC on a diol column (see above) using nhexane/tertiary butylmethylether $\left(90: 10\right.$; flow rate, $\left.0.75 \mathrm{~mL} \mathrm{~min}^{-1}\right)$ by recording the absorption at $255 \mathrm{~nm}$. The identity of PQ-9 was confirmed by mass spectroscopy and by comparing its UV absorption spectrum to that of PQ-9 isolated from A. thaliana leaves by reversed phase HPLC [40]. The content of PQ-9 was calculated based on the amount of the internal standard (ubiquinone-4) taking into account the different molar extinction coefficients of PQ-9 ( $\varepsilon=$ $152001 \mathrm{~mol}^{-1} \mathrm{~cm}^{-1}$ at $\left.255 \mathrm{~nm}\right)$ and ubiquinone-4 $\left(\varepsilon=14900 \mathrm{~L} \mathrm{~mol}^{-1} \mathrm{~cm}^{-1}\right.$ at $275 \mathrm{~nm} ; \varepsilon=87651$ $\mathrm{mol}^{-1} \mathrm{~cm}^{-1}$ at $255 \mathrm{~nm}$; determined photometrically) [41].

PQ-9, PC-8 and MPC-8 were separated by reversed phase HPLC [40]. Briefly, lipids were injected onto an RP18 column (Eurosphere-100, 250 x $4.6 \mathrm{~mm}$, Knauer, Berlin) with 
gradient elution (100\% methanol from $0-10 \mathrm{~min}$, to $3 \%$ methanol/97 \% isopropanol at $30 \mathrm{~min}$,

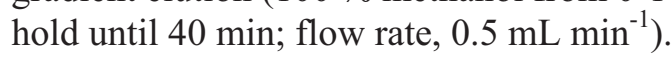

\section{Analysis of quinol lipids by mass spectroscopy}

Quinol lipids were isolated by HPLC as described above and mass spectra recorded using a quadrupole-time of flight mass spectrometer (Q-TOF 6530, Agilent, Waldbronn, Germany). The lipids were dissolved in chloroform/methanol/ammonium acetate (300:665:35) and directly infused at a flow rate of $1 \mu 1 \mathrm{~min}^{-1}$ using a chip-based nanospray ion source (HPLC Chip/MS 1200 with infusion chip, Agilent, Waldbronn, Germany). Samples were analyzed in positive mode with a fragmentor voltage of $270 \mathrm{~V}$. Molecular ions were selected in the quadrupole, fragmented in the collision cell with nitrogen gas and a collision energy of 33 $\mathrm{V}$ ( $\gamma$-tocopherol) and $40 \mathrm{~V}$ (PC-8). Data were processed with the Mass Hunter Workstation software (Version B.02.00; Agilent technologies, Inc 2008).

\section{Fractionation of $A$. thaliana chloroplasts}

Chloroplasts were isolated from leaves of 6 or 8 week old $A$. thaliana plants, as indicated, hypotonically ruptured and subplastidial compartments isolated by centrifugation using a standard sucrose density gradient [15]. The different subfractions were pooled into five fractions according to Western blot analysis using antibodies against plastoglobulin 35 (AtPGL35, plastoglobule marker), translocon at the outer envelope membrane 75 (AtTOC75, envelope marker) and chlorophyll a binding protein ( $\mathrm{CAB}$, thylakoid marker). The gradient fractions F1 and F2 contained mostly plastoglobules, F3 envelopes, and F4 and F5 thylakoids [15]. Tocochromanols were extracted from the five fractions with chloroform/methanol (2:1), and quantified by fluorescence HPLC.

\section{Subcellular localization of VTE4 using green fluorescent protein}

The coding region of VTE4 was amplified from the cDNA clone RAFL07-18-C14 (RIKEN) using the primers PD590 (5'-AGG GAT CCA ATG AAA GCA ACT CTA GCA G) and PD591 (5'-ATC CAT GGA GAG TGG CTT CTG GCA AGT G) adding flanking BamHI and $\mathrm{NcoI}$ restriction sites. The PCR product was cloned into pCL60, 5' and in frame to the green fluorescent protein (GFP) sequence under control of the 35S promoter [42]. The other GFP constructs (PGL35-GFP, AtTIC110-GFP, pSSU-GFP) were described previously [15, 43]. A. thaliana leaves were transiently transformed by particle bombardment as follows: Gold particles $(1.0 \mu \mathrm{m}$, BioRad; $1.5-3 \mu \mathrm{m}$, Aldrich) were sterilized in ethanol and re-suspended in water to a final concentration of $60 \mu \mathrm{g} / \mu \mathrm{L}$. Plasmid DNA $(5 \mu \mathrm{g})$ was precipitated on the gold carrier (50 $\mu \mathrm{L}$ aliquots consisting of a 1:1 mixture of microcarrier from BioRad and Aldrich) by adding $50 \mu \mathrm{L}$ of $2.5 \mathrm{M} \mathrm{CaCl}_{2}$ and $20 \mu \mathrm{l}$ of $0.1 \mathrm{M}$ spermidine free base under continuous vortexing. After washing with ethanol, DNA-coated gold particles were suspended in ethanol, spread on 4 macrocarrier discs (BioRad) and used for ballistic transformation. Leaves of mature $A$. thaliana plants grown on soil for 4 to 7 weeks were placed upside-down on $0.5 \times \mathrm{MS}$ medium and bombarded with a PDS-1000/He BIOLISTIC R Particle Delivery System (BioRad) operating at 1100 PSI He pressure. 24 to $48 \mathrm{~h}$ after transformation, leaves were placed onto glass slides with lanolin. Transient transformation of protoplasts was done with polyethyleneglycol [44], but reducing cellulase and macerozyme (Serva) contents to $1 \%$ and $0.25 \%(\mathrm{w} / \mathrm{v})$, respectively. Fluorescence in transiently transformed leaves and protoplasts was monitored $48 \mathrm{~h}$ after transformation by confocal laser scanning microscopy (LEICA TCS 4D microscope). The FITC (488 nm) laser line was used to detect GFP fluorescence, and chlorophyll autofluorescence was monitored using the TRITC $(568 \mathrm{~nm})$ excitation wavelength.

\section{Electron microscopy}


Chloroplast ultrastructures of four week-old wild type, vte1 and WT-VTE1\#40 A. thaliana plants were examined by electron microscopy. Leaves were fixed in $1 \%$ glutaraldehyde in $0.05 \mathrm{M}$ phosphate buffer overnight at $4^{\circ} \mathrm{C}$, and postfixed in osmium tetroxide for $2 \mathrm{~h}$ at room temperature. After dehydration and embedding in LRWhite resin, ultra-thin sections $(80-90 \mathrm{~nm}$ ) were obtained on a Reichert Ultracut S microtome and mounted on copper grids. Ultra-thin sections were poststained with uranyl acetate and lead citrate and observed with a Philips CM 100 transmission electron microscope at $60 \mathrm{kV}$. Chloroplast area and PG diameter were measured using the ImageJ software.

\section{RESULTS}

\section{Accumulation of PC-8 in $A$. thaliana plants affected in expression of genes of tocopherol synthesis}

Plastochromanol-8 (PC-8), an unusual prenyl quinol lipid, was originally identified in leaves of rubber trees [21] and later, in the seeds of other plant species [22]. To address the question of its distribution, we analyzed different additional plants for the occurrence of PC-8. PC-8 was detected in leaves of tobacco, rice, pumpkin, potato, tomato and Lotus japonicus and in tomato fruits in varous amounts (data not shown). This suggests that PC-8 is widely distributed in the plant kingdom, and it occurs both in monocot and dicot plants and in different plant organs. The occurrence of PC-8 in A. thaliana WT leaves was confirmed by co-elution with PC-8 from linseed oil in fluorescence HPLC at $23 \mathrm{~min}$ (diol column) (Fig. 2A, top panel). PC-8 in A. thaliana leaves constitutes about $1.2 \mathrm{nmol} \mathrm{g}^{-1} \mathrm{FW}$ (equivalent to $5-10 \mathrm{~mol} \%$ of total tocochromanols, i.e. all tocopherols and PC-8) depending on growth conditions and age (Fig. 2A, top panel; Table 1). Therefore, the abundance of PC-8 in leaves is in the range of that of $\gamma$-tocopherol, indicating that it represents an important form of tocochromanols in leaves. Due to the very high content of $\gamma$-tocopherol in WT seeds (ca. $1200 \mathrm{nmol} \mathrm{g}^{-1} \mathrm{FW}$ ), the amount of PC-8 (ca. $13 \mathrm{nmol} \mathrm{g}^{-1} \mathrm{FW}$ ) was equivalent to only $1 \mathrm{~mol} \%$ of total tocochromanols (Table $1)$.

Previously, overexpression of tocopherol cyclase (VTE1) under control of the $35 \mathrm{~S}$ promoter in A. thaliana leaves was reported to result in a strong increase of tocochromanols [34]. After separation of tocochromanols from the VTE1 overexpressing line WT-VTE1\#40 by diol column HPLC [39], it became clear that the PC-8 peak was drastically increased while the $\gamma$-tocopherol peak remained similar to WT (Fig. 2A, middle panel). High amounts of PC-8 were also found in leaves of a second line overexpressing VTE1 (vtel-VTE1\#1; Table 1). The amounts of individual tocopherols in leaves of WT-VTE1\#40 and vtel-VTE1\#1 were only slightly increased (Table 1). In addition to the increase of PC-8 in leaves of lines WT-

VTE1\#40 and vtel-VTE1\#1, PC-8 also accumulated in the seeds of the transgenic lines (Table $1)$.

To unambiguously identify PC-8 in A. thaliana leaves, fractions containing PC-8 were collected from HPLC and subjected to mass spectrometry via quadrupole time of flight (QTOF) mass spectrometry. Fig. 3 shows the mass spectra of PC-8 samples collected from linseed oil and A. thaliana WT-VTE1\#40, and $\gamma$-tocopherol collected from WT leaves. All quinol lipids were ionized as cation radicals $[\mathrm{M}]^{+}$. The cation radical for PC-8 from line WTVTE1\#40 was detected at $750.6294 \mathrm{~m} / \mathrm{z}$ in agreement with the calculated mass of 750.6309 $\mathrm{m} / \mathrm{z}\left(\mathrm{C}_{53} \mathrm{H}_{82} \mathrm{O}_{2}\right)$. After selecting the molecular ions in the quadrupole and fragmentation by collision-induced dissociation, the mass spectra of PC-8 and $\gamma$-tocopherol were recorded. All quinol lipids showed peaks at m/z 151.07 and 191.10 which is in good agreement with the calculated masses of two fragments derived from the PC- $8 / \gamma$-tocopherol head groups (also see: $[25,45])$.

Tocopherol and PQ-9/PC-8 synthesis are closely linked because the two biosynthetic pathways depend on the availability of a common precursor molecule, homogentisate, and in 
part employ the same biochemical reactions (VTE3, VTE1; Fig. 1). To study the role of the enzymes of tocopherol synthesis in PC-8 production, two $A$. thaliana mutants were selected: The vtel mutant carrying a null mutation in tocopherol cyclase [7,46], and two alleles of vte 2 affected in homogentisate phytyltransferase (VTE2; Sattler et al., 2004): vte2-1 contains very low amounts of tocopherol [32] due to an amino acid exchange in VTE2, and the T-DNA mutant vte2-3 is completely devoid of tocopherol (this study). Tocochromanols were absent from vtel leaves or seeds (Table 1). This is in agreement with the scenario that PC-8 synthesis depends on VTE1. Leaves and seeds of vte 2-1 and of vte2-3 contained low amounts of PC-8 similar to WT, indicating that PC-8 synthesis is independent of VTE2 (Fig 3A, B). Therefore, although the vte2-3 mutant is devoid of tocopherol, it still contains PC-8 as a residual form of tocochromanol.

The amount of tocopherol increases in leaves during abiotic stress, e.g. high light, and during senescence $[4,7,47,48]$. The accumulation of tocopherol during stress is correlated with an increase in expression of tocopherol synthesis genes, e.g. HPPD, VTE2 and VTE1 [34, 47]. Table 2 shows that the amount of PC- 8 also accumulates in leaves of $A$. thaliana plants exposed to high light. The proportion of $\mathrm{PC}-8$ relative to total tocochromanols during high light remains unchanged.

Quantification of plastoquinol-9 in A. thaliana lines altered in tocochromanol metabolism

The synthesis of PC- 8 by VTE1 depends on the availability of the precursor PQ-9.

Therefore, the accumulation of PC-8 in VTE1 overexpression lines and the increase during light stress might result in a depletion of the PQ-9 pool in the chloroplasts. PQ-9 was quantified by HPLC in different $A$. thaliana lines (Table 2). PQ-9 content was not altered in the vtel and vte 2 mutants, indicating that a decrease in tocopherol or PC-8 does not affect PQ-9 synthesis. Similar to tocopherol, PQ-9 was increased under high light. In the lines WT-

VTE1\#40 and vtel-VTE1\#1, PQ-9 content was slightly decreased (Table 2). The changes of PQ-9 and PC-8 were recorded in separate HPLC experiments because the separation conditions were optimized for tocochromanols or PQ-9, respectively (Table 2; see methods). However, PQ-9 can also be measured via UV absorption during chromatography of tocochromanols which are recorded by fluorescence. Thus, the ratios of peak areas of PQ-9 (measured by UV absorption) and of PC-8 (measured by fluorescence) were recorded simultaneously in one HPLC experiment. The ratios of PQ- 9 to PC-8 peak areas are drastically decreased upon VTE1 overexpression when measured in the yery same chromatogram $(2.12 \pm 0.67,0.20 \pm 0.08$ and $0.12 \pm 0.05$ for WT, WT-VTE $1 \# 40$ and vte1-VTE1\#1, respectively; $n=3$, mean \pm SD). This is in good accordance with the relative changes in PC-8 and PQ-9 measured in individual chromatograms as shown in Table 2 . In conclusion, the strong accumulation of PC-8 in VTE1 overexpressing lines resulted in a decrease in PQ-9 content which is in agreement with the role of PQ-9 as a precursor for PC-8 synthesis.

\section{Plastochromanol-8 and plastoquinol-9 accumulate in thylakoids and plastoglobules of $A$. thaliana chloroplasts}

Most tocopherol synthesis enzymes localize to the envelope membranes of chloroplasts, while tocopherol cyclase (VTE1) was found in the plastoglobules $[12,14,15]$. To study the localization of PC-8 experimentally, different fractions were isolated from ruptured chloroplasts of 8 week old plants by sucrose density centrifugation. The gradient subfractions were pooled into five fractions enriched in plastoglobules (F1, F2), envelope membranes (F3) and thylakoids (F4, F5) according to the distribution of marker proteins as visualized by immunoblot analysis (Fig. 4A) [15]. Tocochromanols were measured by fluorescence HPLC and calculated as nmol per ml fraction volume [39]. The amount of PC-8 in each of the five fractions was obtained after multiplication with the fraction volume. A large proportion of plastidial PC-8 localizes to plastoglobules (fractions F1 and F2) while the remainder is mostly 
associated with thylakoids (F4 and F5) (Fig. 4B, top panel). The amount of PC-8 in envelope membranes is very low. The tocochromanol distribution in plants overexpressing VTE1 (line WT-VTE1\#40) was assessed to study the subplastidial compartimentation of the extra amount of PC-8 accumulating in the transgenic line. As shown in Fig. 4B (bottom panel), the content of PC-8 relative to the other tocochromanols remained similar in all chloroplast fractions. A large fraction of PC-8 accumulated in the plastoglobule fractions F1 and F2, and the amount of PC-8 associated with thylakoids (F4, F5) was also increased (Fig. 4B).

To study the subplastidial distribution of the PC-8 precursor, PQ-9 was also quantified by HPLC in the chloroplast sucrose gradient fractions from WT. Fig. 4B (middle panel) shows that a large proportion of total PQ-9 is associated with plastoglobules and with thylakoids.

PC-8 was previously shown to harbor antioxidant activity comparable to that of tocopherols [26]. Therefore, the accumulation of PC-8 in the transgenic line WT-VTE1\#40 might contribute to the antioxidant activity of leaves. To analyze the effect of overexpression of VTE1 on photosynthesis, the two lines WT-VTE1\#40 and vtel-VTE1\#1 were exposed to high light for 4 days and employed for the measurement of chlorophyll fluorescence [49]. Quantum yield at normal and high light conditions in the PC-8 accumulating plants was similar to WT (data not shown), indicating that PC-8 accumulation had no major impact on photosynthesis under normal or high light at ambient temperature.

\section{Chloroplast ultrastructure in PC-8 accumulating $A$. thaliana lines}

The fact that PC-8 accumulates in plastoglobules (Fig. 4) suggested that plastoglobule number or size might be altered in chloroplasts after PC-8 accumulation. Analysis by transmission electron microscopy revealed that leaves of four weeks old plants accumulating PC-8 (WT-VTE1\#40) showed a high increase in plastoglobule number (Fig. 5A, bottom panel). While the diameter of the individual plastoglobules remained similar to WT, the number of plastoglobules per chloroplast cross-sectional area in WT-VTE1\#40 $(2.60 \pm 0.30)$ was strongly increased as compared to WT $(1.05 \pm 0.10)$, and this number was significantly reduced in the vtel mutant $(0.80 \pm 0.08)(\mathrm{n}=20$ chloroplasts analyzed for each line). In line WT-VTE1\#40, PC-8 increases to $68 \mathrm{nmol} \mathrm{g}^{-1} \mathrm{FW}$, while the content of its precursor, PQ-9 (20 $\left.\mathrm{nmol} \mathrm{g}{ }^{-1} \mathrm{FW}\right)$, is only slightly reduced as compared to WT (24 nmol g$\left.{ }^{-1} \mathrm{FW}\right)$ (Table 2). Therefore, the increase in plastoglobule number presumably originates from the accumulation of PC-8. However, it is also possible that the accumulation of the VTE1 protein itself affects plastoglobule size because VTE1 also localizes to the plastoglobules.

The plastoglobules in WT-VTE1\#40 are organized in grape-like clusters and localize to the stroma in proximity to the thylakoid membranes, suggesting that they are interconnected (Fig. 5A, bottom panel; see: [14]). The number of plastoglobules in clusters was strongly increased in line WT-VTE1\#40. While WT and vtel contained single plastoglobules or clusters of 2 or 3 plastoglobules, line WT-VTE1\#40 contained clusters containing up to 17 plastoglobules (Fig. 5B). The count of plastoglobules grouped in a cluster was done on twodimensional images of ultrathin-sections likely under-estimating the actual number of plastoglobules per cluster.

\section{Overexpression of $\gamma$-tocopherol methyltransferase (VTE4) in PC-8 accumulating plants}

PC-8 and $\gamma$-tocopherol each lack one methyl group as compared to the head group of $\alpha$ tocopherol which contains three methyl groups (Fig. 1). $\gamma$-Tocopherol is converted to $\alpha$ tocopherol by $\gamma$-tocopherol methyltransferase (VTE4) [8]. The fact that PC-8 occurs in the nonmethylated form in WT and WT-VTE1\#40 suggested that VTE4 activity is limiting or that this enzyme is not specific for PC-8. Therefore, VTE4 was co-overexpressed in line WT-VTE1\#40, and double transgenic lines (WT-VTE1-VTE4) selected by HPLC screening for alterations in tocochromanol composition. In the double transgenic line WT-VTE1-VTE4, the peak for PC-8 in diol column HPLC was strongly reduced with a concomitant increase in the $\alpha$-tocopherol 
peak as compared to the parental line WT-VTE1\#40 (Fig. 2A, bottom panel). Because methylation of PC-8 in line WT-VTE1-VTE4 was expected to result in the accumulation of 5methyl-PC-8 (MPC-8), it was considered that $\alpha$-tocopherol and MPC-8 might co-elute during HPLC analysis on the diol column. Therefore, the peak eluting at 9 min in diol column HPLC of WT-VTE1-VTE4 (Fig. 2A, bottom panel) was collected and separated by reversed phase HPLC. It became clear that this peak contains two substances, $\alpha$-tocopherol, and a second, nonpolar compound eluting at 32 min tentatively identified as MPC-8 (data not shown). Direct injection of a WT-VTE1-VTE4 leaf extract onto reversed phase HPLC confirmed this result, because it revealed the presence of two peaks co-eluting at $32 \mathrm{~min}$ (MPC-8) and PC-8 (33 min) (Fig. 2B). The identity of the PC-8 peak eluting at 33 min on reverse phase HPLC was confirmed by co-elution with authentic PC-8 isolated from linseed via diol column HPLC (data not shown).

MPC-8 was isolated from line WT-VTE1-VTE4 via diol column HPLC and subjected to Q-TOF mass spectrometry in positive mode (Supplemental Figure 1). MPC-8 was ionized as radical cation with a mass of $764.7166 \mathrm{~m} / \mathrm{z}$ in agreement with the calculated mass of 764.6466 $\mathrm{m} / \mathrm{z}\left(\mathrm{C}_{54} \mathrm{H}_{84} \mathrm{O}_{2}\right)$. After fragmentation, a prominent peak at $\mathrm{m} / \mathrm{z} 382.3086$ appeared that can be explained by a cleavage of the molecular ion into two fragments of $382.2866\left(\mathrm{C}_{26} \mathrm{H}_{38} \mathrm{O}_{2}\right)$ and $382.3594\left(\mathrm{C}_{28} \mathrm{H}_{46}\right)$. The peak at 165.0848 which can also be observed in the $\alpha$-tocopherol spectrum is derived from a headgroup fragment $\left(\mathrm{C}_{10} \mathrm{H}_{13} \mathrm{O}_{2}, 165.0916 \mathrm{~m} / \mathrm{z}\right)$.

To study the impact of PC-8 and MPC-8 accumulation on the amount of the precursor molecule, PQ-9 was measured in leaves of the transgenic line WT-VTE1-VTE4. In analogy to the single overexpression lines WT-VTE1\#40 and vte1-VTE1\#1, the amount of PQ-9 in line WT-VTE1-VTE4 was decreased, however to an even stronger extent (Table 2). Taken together, these data strongly suggest that overexpression of VTE4 in line WT-VTE1\#40 results in the partial conversion of PC-8 to MPC-8 via methylation.

A possible explanation for the low degree of PC-8 methylation in leaves might be the localization of the corresponding enzymes to different subplastidial compartments. While the VTE1 protein was previously localized to the plastoglobules of $A$. thaliana $[12,15]$, Soll et al. [2] suggested that the $\gamma$-tocopherol methyltransferase (VTE4) activity is associated with the envelope membranes of spinach chloroplasts. To study the localization of $A$. thaliana VTE4, a VTE4 fusion protein with the green fluorescent protein (GFP) was transiently expressed in leaves, and fluorescence analyzed by confocal microscopy (Fig. 6). VTE4-GFP fluorescence was observed as a peripheral ring around the chloroplasts (Fig. 6A, top panel). This pattern was similar to the GFP fusion of the envelope membrane marker protein TIC110, but different from that of PGL35 and VTE1 which both localize to plastoglobules and show a dotted pattern inside the chloroplasts (Fig. 6A) [15]. Transient expression of the VTE4-GFP fusion protein in A. thaliana protoplasts also revealed a ring-like pattern of fluorescence surrounding the chloroplasts (Fig. 6B). Western blotting using anti-GFP antibodies demonstrated the expression of the VTE4-GFP fusion protein with the correct size in the protoplast expression system. These data suggest that the VTE4 protein localizes to the envelope membranes in accordance with the localization of $\gamma$-tocopherol methyltransferase activity previously determined [2]

\section{DISCUSSION}

\section{Accumulation of PQ-9 and PC-8 in plastoglobules}

Fig. 4 suggests that considerable proportions of the plastidial pools of PQ-9 and PC-8 are associated with plastoglobules. The separation of plastidial subfactions by sucrose density centrifugation was followed by analyzing marker proteins via Western blotting (Fig. 4A). The individual plastidial compartments (plastoglobules, envelopes, thylakoids) are distributed across several gradient fractions which is reflected by the accumulation of marker proteins in 
the different fractions. For example, PGL35 and VTE1 which are considered as markers for plastoglobules are enriched in fractions F1, F2 and F3, but also occur in F4 and F5, albeit at lower level. The presence of these two proteins in fractions F4 and F5 might indicate that they also occur in thylakoids, or that some of the plastoglobules remain connected to the thylakoids, possibly through membraneous filaments [50], and thus are co-isolated with thylakoid fractions. The low levels of the thylakoid marker CAB in F1 and F2 suggest that the prenyl quinols found in these fractions originate from plastoglobules per se, rather than from thylakoid contamination. Thus, although an exact quantification of the lipid distribution is not possible, these data suggest that the quinol lipids PC-8 and PQ-9 are enriched in thylakoid and plastoglobules of chloroplasts $[15,51]$. This finding is in full agreement with results previously obtained for tocopherols, PQ-9 and phylloquinol [15, 20, 51, 52].

The proportions of the two prenyl quinol lipids in the plastoglobules relative to the thylakoids might depend on plant development, because only relatively low numbers of plastoglobules were found in chloroplasts of younger, four weeks old leaves (Fig. 5A), and the plastoglobule number and size is known to increase in older leaves and during stress or senescence. For this reason, the amount of PQ-9 and PC-8 in the plastoglobule fraction might correlate with the number of plastoglobules per chloroplast, and therefore with plant age, development and stress. We obtained similar distributions of PC-8 or PQ-9 to the different subplastidial fractions in chloroplasts isolated from 6 or 8 week old plants (data not shown). Lower proportions of prenyl quinols might be found in leaves from younger plants or plants grown in tissue culture, since these leaves are likely to contain fewer plastoglobules (e.g.[53]). The pool of PQ-9 in plastoglobules not associated with thylakoids might provide a reservoir for antioxidant molecules and electron carriers for photosynthetic electron transport. It is known that the numbers of photosystem II as well as PQ-9 in chloroplasts are present in excess and therefore are not limiting for photosynthesis [54].

\section{Accumulation of PC-8 in WT and in overexpressing plants}

PC-8 was first discovered in rubber tree leaves (Hevea brasiliensis) more than 40 years ago [21]. Since that time, PC-8 was identified in the seeds of a number of plant species [22-24, $27,28,31,39]$. However, reports on the occurrence of PC-8 in plant leaves remained scarce. PC-8 was not measured in the recent studies on tocopherol synthesis and function in $A$. thaliana leaves $[7,46,55,56]$. The two tocochromanols, PC-8 and $\gamma$-tocopherol, are difficult to separate because they share the same head group and therefore, have very similar physical characteristics. During normal phase HPLC, PC-8 can co-elute with $\gamma$-tocopherol, while in reverse phase HPLC, PC-8 is eluted under highly non-polar conditions at the end of the gradient (Fig. 2). Using diol column HPLC [39], all four forms of tocopherol and PC-8 can be separated and quantified. Previous studies and results presented here clearly demonstrate that PC-8 represents an authentic form of tocochromanol in leaves, seeds and other organs of plants including $A$. thaliana in considerable amounts of ca. $5-10 \mathrm{~mol} \%$ of total tocochromanols. Previously, overexpression of VTE1 in A. thaliana leaves was found to result in an apparent increase in the $\gamma$-tocopherol peak in normal phase HPLC [34]. Re-evaluation of the tocochromanol composition of line WT-VTE1\#40 by diol column HPLC revealed that this peak contains two compounds, $\gamma$-tocopherol and PC-8 (Table 1). Thus, it is now clear that VTE1 overexpression in $A$. thaliana leaves causes a drastic increase in PC-8 content from ca. 1 to $70 \mathrm{nmol} \mathrm{g}{ }^{-1} \mathrm{FW}$, such that PC-8 becomes the most abundant tocochromanol. The amounts of the individual tocopherol forms were only slightly increased in the leaves of the two lines WTVTE1 \#40 and vtel-VTE1\#1 (Table 1; [34]). In seeds of transgenic plants, PC-8 also accumulates while the amounts of the different tocopherol forms are comparable to WT levels. Overexpression of VTE1 under control of the $35 \mathrm{~S}$ promoter presumably results in the accumulation of low levels of VTE1 in seeds which can explain the fact that less pronounced tocochromanol changes are observed in seeds. Previous studies demonstrated that seed-specific 
overexpression of corn or $A$. thaliana VTE1 in rape using the napin promoter resulted in a strong accumulation in PC-8 accompanied with moderate increases in other tocopherol forms, particularly $\delta$-tocopherol $[30,31]$. The increase in PC- 8 and tocopherols was observed in lines overexpressing VTE1 as a single construct, or VTE1 in combination with VTE2 or HPPD. In contrast to VTE1, the overexpression of VTE2 in A. thaliana leaves resulted in a much stronger increase in total tocopherols by a factor of 4.4, while PC-8 was not measured [57]. Taken together, these data indicate that VTE2 is limiting for tocopherol accumulation because it is required for the conversion of homogentisate to MPBQ (Fig. 1). The impact of VTE1 overexpression on tocopherol synthesis is much lower due to the limitation in supply of the precursors MPBQ/DMPBQ. However, VTE1 overexpression results in a strong increase in PC8 by cyclization of PQ-9 which itself is present in chloroplasts in high amounts (Table 2).

During a 12 day high light stress experiment, the transcripts of HPPD and VTE2 were found to be strongly upregulated via real-time PCR analysis, while the expression patterns of VTE1 and VTE4 were less affected with a moderate induction for the two latter genes around day three (see Fig. 5 of [47]). It was concluded that HPPD and VTE2 are limiting for tocopherol synthesis during high light stress. On the other hand, Northern blot experiments revealed that VTE1 expression is upregulated during the first four days of high light stress [34]. Expression profiling of a $24 \mathrm{~h}$ time course experiment of abscisic acid treated $A$. thaliana seedlings revealed a coordinated upregulation of expression of several tocopherol synthesis genes, i.e. HPPD, VTE2, VTE1 and VTE4 [58]. Taken together, these data suggest that the increase of tocopherol synthesis during abiotic stress is mediated via the induction of several genes, possibly with different stress-dependent induction kinetics.

\section{Overexpressing VTE4 in VTE1 expressing plants}

In A. thaliana leaves, $\alpha$-tocopherol carrying three methyl groups is the predominant form of tocopherols, indicating that the largest portion of $\gamma$-tocopherol is methylated by VTE4. $\gamma$-Tocopherol and PC-8 both contain two methyl groups in their head group. VTE1 was previously suggested to localize to the plastoglobules of chloroplasts $[12,15]$, while the other enzymes of tocopherol synthesis including VTE4 are mostly associated with the envelope membranes [2]. Transient expression of VTE4-GFP under control of the $35 \mathrm{~S}$ promoter resulted in the accumulation of fluorescence in a ring at the boundary of the chloroplasts indicative for envelope localization (Fig. 6). It is possible that the distribution of tocopherols between plastoglobules, thylakoids and envelopes is in a diffusion-dependent equilibrium. Furthermore, it is possible that the enzymes (VTE1, VTE4) involved in the final biosynthetic steps are distributed between the different compartments, i.e. plastoglobules, stroma and envelopes of chloroplasts. Thus, a small amount of VTE4 might localize to plastoglobules or might be mobile, thereby having direct access to $\gamma$-tocopherol for methylation. After strong overexpression of VTE4, a fraction of PC-8 was converted to a compound tentatively identified as MPC-8. This suggests that PC- 8 can be used by VTE4 as a substrate, but with lower activity. Furthermore, these data are in agreement with the fact that VTE4 from tocopherol-containing plants prefers $\gamma$-tocopherol, while VTE4 from tocotrienol-containing species uses $\gamma$-tocotrienol as preferred substrate. [59]. As PC-8 contains an unsaturated side chain similar to $\gamma$-tocotrienol, it is possible that the A. thaliana enzyme is more active with $\gamma$-tocopherol than with PC- 8 . This scenario implies that the rate of conversion of PC- 8 to MPC- 8 is low because PC- 8 is not a good substrate for VTE4.

\section{Prenyl quinol lipids in the vte1 and vte2 mutants}

Results presented here clearly show that the vtel mutant which lacks tocopherol cyclase is not only devoid of tocopherol, as described previously [7, 55], but also lacks PC-8, another tocochromanol form. PC-8 was described to harbor antioxidant activity, comparable to that of tocopherols [26]. Therefore, the additional loss of PC-8 in the vtel mutant should be taken into 
consideration in physiological studies employing this mutant, e.g. [7, 32, 34, 46, 56, 60-62]. Given the fact that PC-8 amounts to only $5-10 \mathrm{~mol} \%$ of total tocochromanols in WT leaves, it is likely that most of the effects observed in vtel mutant plants are indeed caused by the deficiency in tocopherol synthesis per se, rather than by the additional lack of PC-8.

Similarly to vte 1, the vte 2 mutant is totally deficient in tocopherol. Table 1 shows that vte 2 contains an amount of PC-8 very similar to that of WT leaves, i.e. $5-10 \mathrm{~mol} \%$ of tocochromanol in WT. The presence of a residual amount of PC- 8 in vte 2 suggests that this compound might exert some antioxidant functions. Given the fact that the amount of PC- 8 is rather low, it is likely that the physiological effects observed in vte 2 mutant plants predominantly originate from tocopherol deficiency [32, 46, 56, 61]. However, it should be considered that vte 2 plants do contain PC-8, and this might partially suppress some physiological effects of tocopherol deficiency (e.g. [32]).

\section{ACKNOWLEDGMENTS}

We would like to thank Lutz Neumetzler, Nicolai Obel and Markus Pauly (Max Planck Institute of Molecular Plant Physiology, Golm) for help with mass spectroscopic analyses of tocochromanols, and Michèle Vlimant (University of Neuchâtel, Laboratory of Animal Physiology) for help with electron microscopy. We would also like to acknowledge support by SystemsX PGCE.

\section{FUNDING}

This work was in part funded by a grant of the Deutsche Forschungsgemeinschaft [DFG, Do520/7-1]. C.B. and F.K. are supported by University of Neuchâtel, the Swiss National Center for Competence in Research [NCCR,"Plant Survival"] and Swiss National Science Foundation [SNF grant 3100A0-109667\} 


\section{REFERENCES}

1 Food and Nutrition Board/Institute of Medicine. (2003) 6. Vitamin E. In Dietary reference intakes for vitamin C, vitamin E, selenium and carotenoids. pp. 186-283, Natl Acad Press, Washington, DC

2 Soll, J., Schultz, G., Joyard, J., Douce, R. and Block, M. A. (1985) Localization and synthesis of prenylquinones in isolated outer and inner envelope membranes from spinach chloroplasts. Arch Biochem Biophys. 238, 290-299

3 Collakova, E. and DellaPenna, D. (2001) Isolation and functional analysis of homogentisate phytyltransferase from Synechocystis sp PCC 6803 and Arabidopsis. Plant Physiol. 127, 1113-1124

4 Motohashi, R., Ito, T., Kobayashi, M., Taji, T., Nagata, N., Asami, T., Yoshida, S., Yamaguchi-Shinozaki, K. and Shinozaki, K. (2003) Functional analysis of the $37 \mathrm{kDa}$ inner envelope membrane polypeptide in chloroplast biogenesis using a Ds-tagged Arabidopsis pale-green mutant. Plant Journal. 34, 719-731

5 Cheng, Z., Sattler, S., Maeda, H., Sakuragi, Y., Bryant, D. A. and DellaPenna, D. (2003) Highly divergent methyltransferases catalyze a conserved reaction in tocopherol and plastoquinone synthesis in cyanobacteria and photosynthetic eukaryotes. Plant Cell. 15, 2343-2356

6 Van Eenennaam, A. L., Lincoln, K., Durrett, T. P., Valentin, H. E., Shewmaker, C. K., Thorne, G. M., Jiang, J., Baszis, S. R., Levering, C. K., Aasen, E. D., Hao, M., Stein, J. C., Norris, S. R. and Last, R. L. (2003) Engineering vitamin E content: from Arabidopsis mutant to soy oil. Plant Cell. 15, 3007-3019

7 Porfirova, S., Bergmüller, E., Tropf, S., Lemke, R. and Dörmann, P. (2002) Isolation of an Arabidopsis mutant lacking vitamin E and identification of a cyclase essential for all tocopherol biosynthesis. Proc Natl Acad Sci U S A. 99, 12495-12500

8 Shintani, D. and DellaPenna, D. (1998) Elevating the vitamin E content of plants through metabolic engineering. Science. 282, 2098-2100

9 Grusak, M. A. and DellaPenna, D. (1999) Improving the nutrient composition of plants to enhance human nutrition and health. Annu. Rev. Plant Physiol. Plant Mol. Biol. . 50, 133-161

10 DellaPenna, D. (2005) Progress in the dissection and manipulation of vitamin E synthesis. Trends in Plant Science. 10, 574-579

11 Dörmann, P. (2007) Functional diversity of tocochromanols in plants. Planta. 225, 269275

12 Ytterberg, A. J., Peltier, J. B. and van Wijk, K. J. (2006) Protein profiling of plastoglobules in chloroplasts and chromoplasts. A surprising site for differential accumulation of metabolic enzymes. Plant Physiol. 140, 984-997

13 Bréhélin, C., Kessler, F. and van Wijk, K. J. (2007) Plastoglobules: versatile lipoprotein particles in plastids. Trends in Plant Science. 12, 260-266

14 Austin, J. R., 2nd, Frost, E., Vidi, P. A., Kessler, F. and Staehelin, L. A. (2006) Plastoglobules are lipoprotein subcompartments of the chloroplast that are permanently coupled to thylakoid membranes and contain biosynthetic enzymes. Plant Cell. 18, 1693-1703

15 Vidi, P. A., Kanwischer, M., Baginsky, S., Austin, J. R., Csucs, G., Dormann, P., Kessler, F. and Brehelin, C. (2006) Tocopherol cyclase (VTE1) localization and vitamin E accumulation in chloroplast plastoglobule lipoprotein particles. J Biol Chem. 281, 11225-11234

16 Norris, S. R., Barrette, T. R. and Dellapenna, D. (1995) Genetic dissection of carotenoid synthesis in Arabidopsis defines plastoquinone as an essential component of phytoene desaturation. Plant Cell. 7, 2139-2148 
17 Sadre, R., Gruber, J. and Frentzen, M. (2006) Characterization of homogentisate prenyltransferases involved in plastoquinone- 9 and tocochromanol biosynthesis. FEBS Lett. 580, 5357-5362

18 Venkatesh, T. V., Karunanandaa, B., Free, D. L., Rottnek, J. M., Baszis, S. R. and Valentin, H. E. (2006) Identification and characterization of an Arabidopsis homogentisate phytyltransferase paralog. Planta. 223, 1134-1144

19 Loll, B., Kern, J., Saenger, W., Zouni, A. and Biesiadka, J. (2005) Towards complete cofactor arrangement in the $3.0 \AA$ resolution structure of photosystem II. Nature. 438 , 1040-1044

20 Tevini, M. and Steinmüller, D. (1985) Composition and function of plastoglobuli. II. Lipid composition of leaves and plastoglobuli during beech leaf senescence. Planta. 163, 91-96

21 Whittle, K. J., Dunphy, P. J. and Pennock, J. F. (1965) Plastochromanol in the leaves of Hevea brasiliensis. Biochem. J. 96, 17c-19c

22 Thompson, J. N. and Hatina, G. (1979) Determination of tocopherols and tocotrienols in foods and tissues by high performance lipid chromatography. J Liq Chromatogr. 2, 327-344

23 Dunphy, P. J., Whittle, K. J. and Pennock, J. F. (1966) Plastochromanol. In Biochemistry of chloroplasts (Goodwin, T. W., ed.).pp. 165-171, Academic Press, London

24 Leerbeck, E., Søndergaard, E. and Dam, H. (1967) Occurrence of a plastochromanol in linseed oil. Acta Chem. Scand. 21, 2582

25 Mayer, H., Metzger, J. and Isler, O. (1967) 139. Über die Chemie des Vitamins E. Die Sterochemie von natürlichem $\gamma$-Tocotrienol (Plastochromanol-3), Plastochromanol-8 und Plastochromenol-8. Helv. Chim. Acta. 50, 1376-1393

26 Olejnik, D., Gogolewski, M. and Nogala-Kałucka, M. (1997) Isolation and some properties of plastochromanol-8. Nahrung. 41, 101-104

27 Bagci, E. and Karaagacli, Y. (2004) Fatty acid and tocochromanol patterns of Turkish pines. Acta Biologica Cracoviensia Series Botanica. 46, 95-100

28 Kriese, U., Schumann, E., Weber, W. E., Beyer, M., Brühl, L. and Matthäus, B. (2004) Oil content, tocopherol composition and fatty acid patterns of the seeds of 51 Cannabis sativa L. genotypes. Euphytica. 137, 339-351

29 Matthäus, B. and Özcan, M. (2005) Glucosinolates and fatty acid, sterol, and tocopherol composition of seed oils from Capparis spinosa var. spinosa and Capparis ovata Desf. var. canescens (Coss.) Heywood. J Agricult Food Chem. 53, 7136-7141

30 Raclaru, M., Gruber, J., Kumar, R., Sadre, R., Lühs, W., Zarhloul, M., Friedt, W., Frentzen, M. and Weier, D. (2006) Increase of the tocochromanol content in transgenic Brassica napus seeds by overexpression of key enzymes involved in prenylquinone biosynthesis. Molec Breeding. 18, 93-107

31 Kumar, R., Raclaru, M., Schusseler, T., Gruber, J., Sadre, R., Luhs, W., Zarhloul, K. M., Friedt, W., Enders, D., Frentzen, M. and Weier, D. (2005) Characterisation of plant tocopherol cyclases and their overexpression in transgenic Brassica napus seeds. FEBS Letters. 579, 1357-1364

32 Havaux, M., Eymery, F., Porfirova, S., Rey, P. and Dörmann, P. (2005) Vitamin E protects against photoinhibition and photooxidative stress in Arabidopsis thaliana. Plant Cell. 17, 3451-3469

33 Rosso, M. G., Li, Y., Strizhov, N., Reiss, B., Dekker, K. and Weisshaar, B. (2003) An Arabidopsis thaliana T-DNA mutagenized population (GABI-Kat) for flanking sequence tag-based reverse genetics. Plant Molecular Biology. 53, 247-259

34 Kanwischer, M., Porfirova, S., Bergmuller, E. and Dormann, P. (2005) Alterations in tocopherol cyclase activity in transgenic and mutant plants of Arabidopsis affect 
tocopherol content, tocopherol composition, and oxidative stress. Plant Physiol. 137, 713-723

35 Höfgen, R. and Willmitzer, L. (1990) Biochemical and genetic analysis of different patatin isoforms expressed in various organs of potato (Solanum tuberosum). Plant Sci. 66, 221-230

36 Clough, S. J. and Bent, A. F. (1998) Floral dip: a simplified method for Agrobacteriummediated transformation of Arabidopsis thaliana. Plant J. 16, 735-743

37 Savidge, B., Weiss, J. D., Wong, Y.-H. H., Lassner, M. W., Mitsky, T. A., Shewmaker, C. K., Post-Beittenmiller, D. and Valentin, H. E. (2002) Isolation and characterization of homogentisate phytyltransferase genes from Synechocystis sp. PCC 6803 and Arabidopsis. Plant Physiol. 129, 321-332

38 Schüep, W. and Rettenmeier, R. (1994) Analysis of vitamin E homologs in plasma and tissue: High-Performance Liquid Chromatography. Meth Enzymol. 234, 294-302

39 Balz, M., Schulte, E. and Thier, H.-P. (1992) Trennung von Tocopherolen und Tocotrienolen duch die HPLC. Fat Sci Technol. 94, 209-213

40 Johnson, T. W., Shen, G., Zybailov, B., Kolling, D., Reategui, R., Beauparlant, S., Vassiliev, I. R., Bryant, D. A., Jones, A. D., Golbeck, J. H. and Chitnis, P. R. (2000) Recruitment of a foreign quinone into the A1 site of photosystem I: I Genetic and physiological characterization of phylloquinone biosynthetic pathway mutants in Synechocystis sp PCC 6803. J Biol Chem. 275, 8523-8530

41 Dawson, R. M. C., Elliott, D. C., Elliott, W. H. and Jones, K. M. (1985) Data for biochemical research. Oxford University Press, Oxford, UK

42 Bauer, J., Hiltbrunner, A., Weibel, P., Vidi, P.-A., Alvarez-Huerta, M., Smith, M. D., Schnell, D. J. and Kessler, F. (2002) Essential role of the G-domain in targeting of the protein import receptor atToc159 to the chloroplast outer membrane. J Cell Biol. 159, $845-854$

43 Inabe, T., Alvarez-Huerta, M., Li, M., Bauer, J., Ewers, C., Kessler, F. and Schnell, D. (2005) Arabidopsis Tic110 is essential for the assembly and function of the protein import machinery of plastids. Plant Cell. 17, 1482-1496

44 Jin, J. B., Kim, Y. A., Kim, S. J., Lee, S. H., Kim, D. H., Cheong, G. W. and Hwang, I. (2001) A new dynamin-like protein, ADL6, is involved in trafficking from the transGolgi network to the central vacuole in Arabidopsis Plant Cell. 13, 1511-1526

45 Cheng, W.-Y., Kuo, Y.-H. and Huang, C.-J. (2007) Isolation and identification of novel estrogenic compounds in yam tuber (Dioscorea alata cv. Tainung No. 2). J Agricult Food Chem. 55, 7350-7358

46 Sattler, S. E., Gilliland, L. U., Magallanes-Lundback, M., Pollard, M. and DellaPenna, D. (2004) Vitamin E is essential for seed longevity and for preventing lipid peroxidation during germination. Plant Cell. 16, 1419-1432

47 Collakova, E. and DellaPenna, D. (2003) The role of homogentisate phytyltransferase and other tocopherol pathway enzymes in the regulation of tocopherol synthesis during abiotic stress. Plant Physiology. 133, 930-940

48 Rise, M., Cojocaru, M., Gottlieb, H. E. and Goldschmidt, E. E. (1989) Accumulation of $\alpha$-tocopherol in senescing organs as related to chlorophyll degradation. Plant Physiology. 89, 1028-1030

49 Schreiber, U., Schliwa, U. and Bilger, W. (1986) Continuous recording of photochemical and nonphotochemical quenching with a new type of modulation fluorometer. Photosynth Res. 10, 51-62

50 Kessler, F., Schnell, D. and Blobel, G. (1999) Identification of proteins associated with plastoglobules isolated from pea (Pisum sativum L.) chloroplasts. Planta. 208, 107-113

51 Lohmann, A., Schöttler, M. A., Bréhélin, C., Kessler, F., Bock, R., Cahoon, E. B. and Dörmann, P. (2006) Deficiency in phylloquinone (vitamin K1) methylation affects 
prenyl quinone distribution, photosystem I abundance and anthocyanin accumulation in the Arabidopsis AtmenG mutant. J Biol Chem. 281, 40461-40472

52 Steinmüller, D. and Tevini, M. (1985) Composition and function of plastoglobuli. I. Isolation and purification from chloroplasts and chromoplasts. Planta. 163, 201-207

53 Matringe, M., Ksas, B., Rey, P. and Havaux, M. (2008) Tocotrienols, the unsaturated forms of vitamin E, can function as antioxidants and lipid protectors in tobacco leaves. Plant Physiol. 147, 764-778

54 Bailey, S., Walters, R., Jansson, S. and Horton, P. (2001) Acclimation of Arabidopsis thaliana to the light environment: the existence of separate low light and high light responses. Planta. 213, 794-801

55 Sattler, S. E., Cahoon, E. B., Coughlan, S. J. and DellaPenna, D. (2003) Characterization of tocopherol cyclases from higher plants and cyanobacteria. Evolutionary implications for tocopherol synthesis and function. Plant Physiology. 132, 2184-2195

56 Maeda, H., Song, W., Sage, T. L. and DellaPenna, D. (2006) Tocopherols play a crucial role in low-temperature adaptation and Phloem loading in Arabidopsis. Plant Cell. 18, 2710-2732

57 Collakova, E. and DellaPenna, D. (2003) Homogentisate phytyltransferase activity is limiting for tocopherol biosynthesis in Arabidopsis. Plant Physiology. 131, 632-642

58 Ghassemian, M., Lutes, J., chang, H.-S., Lange, I., Chen, W., Zhu, T., Wang, X. and Lange, M. (2008) Abscisic acid-induced modulation of metabolic and redox control pathways in Arabidopsis thaliana. Phytochemistry 69, 2899-2911

59 Van Eenennaam, A., Valentin, H. E., Karunanandaa, B., Hao, M., Aasen, E. and Levering, C. (2003) Methyltransferase genes and uses thereof. ed.)^eds.), United States Patent Application 20030154513

60 Collin, V., Eymery, F., Genty, B., Rey, P. and Havaux, M. (2008) Vitamin E is essential for the tolerance of Arabidopsis thaliana to metal-induced oxidative stress. Plant Cell Envr. 31, 244-257

61 Sattler, S. E., Mene-Saffrane, L., Farmer, E. E., Krischke, M., Mueller, M. J. and DellaPenna, D. (2006) Nonenzymatic lipid peroxidation reprograms gene expression and activates defense markers in Arabidopsis tocopherol-deficient mutants. Plant Cell. 18, 3706-3720

62 Maeda, H., Sakuragi, Y., Bryant, D. A. and Dellapenna, D. (2005) Tocopherols protect Synechocystis sp. strain PCC 6803 from lipid peroxidation. [erratum appears in Plant Physiol. 2006 Apr;140(4):1522]. Plant Physiology. 138, 1422-1435 


\section{FIGURE LEGENDS}

\section{Figure 1 Biosynthetic pathways for tocopherols, tocotrienols, plastoquinol-9 and plastochromanol-8}

Homogentisate serves as precursor for the synthesis of tocopherols, tocotrienols and plastoquinol-9 in chloroplasts of plants. In A. thaliana, $\alpha$-tocopherol and $\gamma$-tocopherol are the predominant forms of tocopherol, while $\delta$-tocopherol and $\beta$-tocopherol represent minor forms (not shown). Tocotrienols (e.g., $\gamma$-tocotrienol, "plastochromanol-3") accumulate predominantly in monocotyledonous species (dashed arrows). Plastoquinol-9 is derived from homogentisate by transfer of a solanesyl group and methylation. Formation of a second ring by VTE1 results in plastochromanol-8 (PC-8). In transgenic A. thaliana lines overexpressing VTE1 and VTE4, a novel compound accumulates (5-methyl-plastochromanol-8, MPC-8; dashed arrow). The reactions representing the major pathways in A. thaliana are shaded in grey. DMPBQ, 2,3dimethyl-5-phytyl-1,4-benzoquinol; HPPD, p-hydroxyphenylpyruvate dioxygenase; MPBQ, 2methyl-6-phytyl-1,4-benzoquinol; MGGBQ, 2-methyl-6-geranylgeranyl-1,4-benzoquinol; DMGGBQ, 2,3-dimethyl-5-geranylgeranyl-1,4-benzoquinol; MSBQ, 2-methyl-6-solanesyl1,4-benzoquinol.

\section{Figure 2 Tocochromanol composition in leaves of $A$. thaliana plants altered in VTE1 or VTE4 expression}

Tocochromanols were separated by (A) HPLC on a diol column according to Balz et al. (1992) [39], or (B) by reverse phase HPLC (only the part of the reverse phase chromatogram is shown that contains PC-8 and MPC-8). WT, A. thaliana wild type; WT-VTE1\#40, plants overexpressing VTE1 [34] VTE1-VTE4, plants overexpressing VTE1 and VTE4. Individual peaks were identified using standards for $\alpha-, \beta-, \gamma$ - and $\delta$-tocopherol. PC- 8 was identified by co-elution with PC-8 from a linseed oil standard (not shown). A second chromatography of the peak collected at $23 \mathrm{~min}$ in the diol HPLC chromatogram of WT-VTE1\#40 resulted in a peak with a retention time of $33 \mathrm{~min}$ in reverse phase HPLC indicating that it is PC-8. The peak collected at $8 \mathrm{~min}$ from the diol chromatogram of VTE1-VTE4 gave rise to a peak at $32 \mathrm{~min}$ in reverse phase conditions indicating that it was MPC-8.

Figure 3 Identification of PC-8 by quadrupole-time of flight mass spectrometry (A), Mass spectrum of PC-8 from linseed oil; (B) PC-8 purified from line WT-VTE1\#40; (C) $\gamma$-tocopherol purified from $A$. thaliana WT leaves. Lipids were extracted and purified by diol column HPLC prior to analysis by Q-TOF mass spectrometry via direct infusion. The spectra were obtained by collision-induced dissociation after selecting the molecular ion peak in the quadrupole ( $\mathrm{M}+=750.63$ and 416.36 for PC- 8 and $\gamma$-tocopherol, respectively). The fragmentation patterns for PC- 8 and $\gamma$-tocopherol are also shown. Note that PC- 8 and $\gamma$ tocopherol were ionized as cation radicals $\left[\mathrm{M}^{\bullet+}\right]$.

\section{Figure 4 Plastoquinol-9 and plastochromanol-8 content in chloroplast fractions from $A$. thaliana leaves \\ Chloroplasts were isolated from leaves of 8 week old plants and after ruptured for subsequent isolation of different subplastidial fractions. (A) Immunoblot analysis of chloroplast membrane fractions using antibodies to marker proteins. Chloroplast membranes were separated by ultracentrifugation and 29 fractions of $1 \mathrm{ml}$ collected. Proteins contained in $400 \mu \mathrm{l}$ of fractions $1-17,200 \mu 1$ of fractions $19-23,100 \mu 1$ of fraction 25 and $50 \mu 1$ of fractions 27-29 were precipitated, separated by SDS-PAGE and transferred to nitrocellulose membranes. The membranes were sequentially probed with antisera raised against plastoglobulin AtPGL35 (plastoglobules), VTE1 (plastoglobules), outer envelope membrane protein AtTOC75, inner envelope membrane protein AtTIC110, and chlorophyll A binding protein (thylakoids) and}


pooled accordingly (F1, F2, plastoglobules; F3, envelopes; F4, F5 thylakoids). (B) PC-8 and PQ-9 were measured by HPLC on a diol column and results calculated in nmol prenyl quinol $/ \mathrm{ml}$ fraction. After multiplication with the respective fraction volume, the amounts in the different fractions were calculated as \% of the total prenyl quinol pool present in all sucrose gradient fractions. Data represent mean $\pm \mathrm{SD}$ of three measurements of fractions from one gradient. Similar results were obtained by measuring fractions derived from a separate biological experiment.

Figure 5 Increase in plastoglobule number in VTE1 overexpressing plants

(A) Electron-microscopic analysis of chloroplasts from A. thaliana WT, vte1 mutant and the overexpressing line WT-VTE1\#40. In WT-VTE1\#40, a large number of plastoglobules exists in clusters of two, three or more plastoglobules, oftentimes in grape-like structures. Bars $=1$ $\mu \mathrm{m}$. (B) Number of plastoglobules (in \% of total) occurring in clusters. In WT and vtel, no clusters with more than three plastoglobules were observed. In WT-VTE1 $\# 40$, only $37 \%$ of plastoglobules occur in an isolated form. Clusters of 4 or more plastoglobules are frequent in WT-VTE1\#40. A total of 20 chloroplasts from three different plants were analyzed for each line. Similar results were obtained with line vtel-VTE1\#1.

Figure 6 Subcellular localization of $\boldsymbol{\gamma}$-tocopherol methyltransferase (VTE4) (A) A. thaliana leaf epidermal cells expressing fusion proteins with the green fluorescent protein after biolistic transformation: VTE4-GFP ( $\gamma$-tocopherol methyltransferase), PGL35GFP (plastoglobules), AtTIC110-GFP (inner envelopes), pSSU-GFP (precursor for the RUBISCO small subunit, stroma). (B) Transient expression of VTE4-GFP in A. thaliana protoplasts. Bars $=5 \mu \mathrm{m}$. Fluorescence was observed by confocal microscopy. Chlorophyll, autofluorescence after excitation at $568 \mathrm{~nm}$; GFP, excitation at $488 \mathrm{~nm}$; merge, overlap of chlorophyll and GFP and fluorescence; in $B$, the bright field picture of the protoplast is also shown. (C) Western blot of protoplasts expressing VTE4-GFP. Proteins on the membrane were visualized with amidoblack (lane 1) or with anti-GFP antibodies (lane 2). 
Table 1 Tocochromanol content in $A$. thaliana tocopherol mutants and overexpressing lines

Tocochromanol compositions of leaves and seeds of $A$. thaliana WT, tocopherol mutants (vtel, vte2-1, vte2-3) and plants overexpressing VTE1 (WT-VTE1\#40, vte1-VTE1\#1) were determined by diol column HPLC. Data represent mean \pm SD of three measurements each. n.d., not detectable.

\begin{tabular}{|c|c|c|c|c|c|}
\hline \multirow[t]{2}{*}{ Leaves } & \multicolumn{5}{|c|}{$\alpha$-Tocopherol $\beta$-Tocopherol $\gamma$-Tocopherol $\delta$-Tocopherol $\quad$ PC- 8} \\
\hline & \multicolumn{4}{|c|}{ nmol g ${ }^{-1}$ Fresh Weight } & \\
\hline WT & $25.2 \pm 4.5$ & $0.5 \pm 0.1$ & $3.0 \pm 0.7$ & $0.7 \pm 0.1$ & $1.2 \pm 0.2$ \\
\hline vtel & n.d. & n.d. & n.d. & n.d. & n.d. \\
\hline vte2-1 & $0.4 \pm 0.1$ & n.d. & n.d. & n.c & $2.2 \pm 0.3$ \\
\hline vte2-3 & n.d. & n.d. & n.d. & n.d. & $2.1 \pm 0.4$ \\
\hline WT-VTE1\#40 & $30.7 \pm 4.3$ & $6.1 \pm 2.6$ & $4.1 \pm 0.6$ & $5.7 \pm 0.6$ & $67.9 \pm 13.5$ \\
\hline vtel-VTE1\#1 & $37.8 \pm 5.2$ & $1.0 \pm 0.5$ & $7.2 \pm 2.4$ & $1.9 \pm 0.6$ & $77.2 \pm 10.4$ \\
\hline Seeds & \multicolumn{3}{|c|}{$\alpha$-Tocopherol $\beta$-Tocopherol } & -Tocopherol & $\mathrm{PC}-8$ \\
\hline & & nmolg & Fresh Wei & & \\
\hline WT & $30.9 \pm 1.9$ & n.d. & $1309.2 \pm 23.1$ & $44.9 \pm 3.3$ & $13.5 \pm 1.1$ \\
\hline vtel & n.d. & n.d. & n.d. & n.d. & n.d. \\
\hline vte2-1 & n.d. & n.d. & $22.2 \pm 0.7$ & $1.5 \pm 0.1$ & $7.2 \pm 0.2$ \\
\hline vte $2-3$ & n.d. & n.d. & 0.0 & $0.9 \pm 0.1$ & $19.0 \pm 1.0$ \\
\hline WT-VTE1\#40 & $21.4 \pm 2.1$ & n.d. & $1143.5 \pm 93.0$ & $198.7 \pm 14.6$ & $93.9 \pm 7.4$ \\
\hline vtel-VTE1\#1 & $0.8 \pm 0.2$ & n.d. & $961.1 \pm 62.0$ & $19.0 \pm 1.5$ & $162.0 \pm 6.0$ \\
\hline
\end{tabular}

Table 2 Plastochromanol-8 and plastoquinol-9 contents of $A$. thaliana leaves of tocopherol mutants and overexpressing plants

PC-8 and PQ-9 contents were determined by diol column HPLC. Data represent mean \pm SD of three measurements each. Low (standard) light, plants raised at $120 \mu \mathrm{mol} \mathrm{s} \mathrm{m}^{-1}$; high light, plants exposed to $600 \mu \mathrm{mol} \mathrm{s}^{-1} \mathrm{~m}^{-2}$ for four days; n.d., not detectable.

\begin{tabular}{lccc}
\hline Leaves & \multicolumn{1}{c}{ PC-8 } & PQ-9 & MPC-8 \\
\cline { 2 - 4 } WT & \multicolumn{2}{c}{ nmol g } & Fresh Weight \\
vte1 & $1.2 \pm 0.2$ & $23.8 \pm 3.1$ & n.d. \\
vte1-1 & n.d. & $22.8 \pm 1.5$ & n.d. \\
vte1-3 & $2.2 \pm 0.3$ & $23.8 \pm 2.0$ & n.d. \\
WT-VTE1 440 & $0.2 \pm 0.0$ & $26.2 \pm 3.0$ & n.d. \\
vte1-VTE1\#1 & $77.9 \pm 13.5$ & $19.8 \pm 1.2$ & n.d. \\
WT-VTE1- & & & n.d. \\
VTE4 & $29.2 \pm 7.2$ & $11.9 \pm 2.1$ & $33.5 \pm 5.7$ \\
Low Light & $1.2 \pm 0.2$ & $21.6 \pm 1.9$ & n.d. \\
High Light & $4.0 \pm 0.6$ & $36.9 \pm 2.0$ & n.d. \\
\hline
\end{tabular}




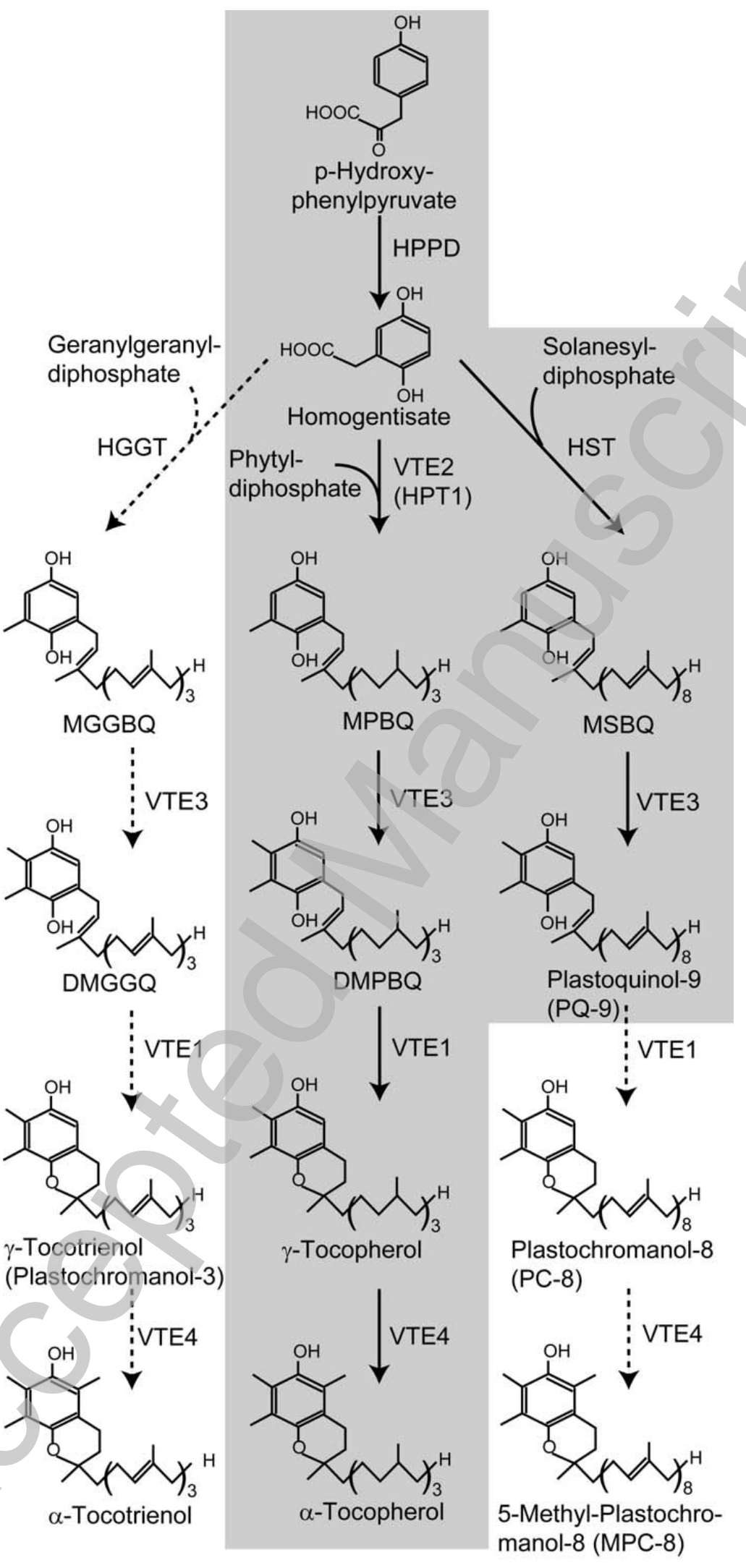

Zbierzak et al. (2009) Figure 1 


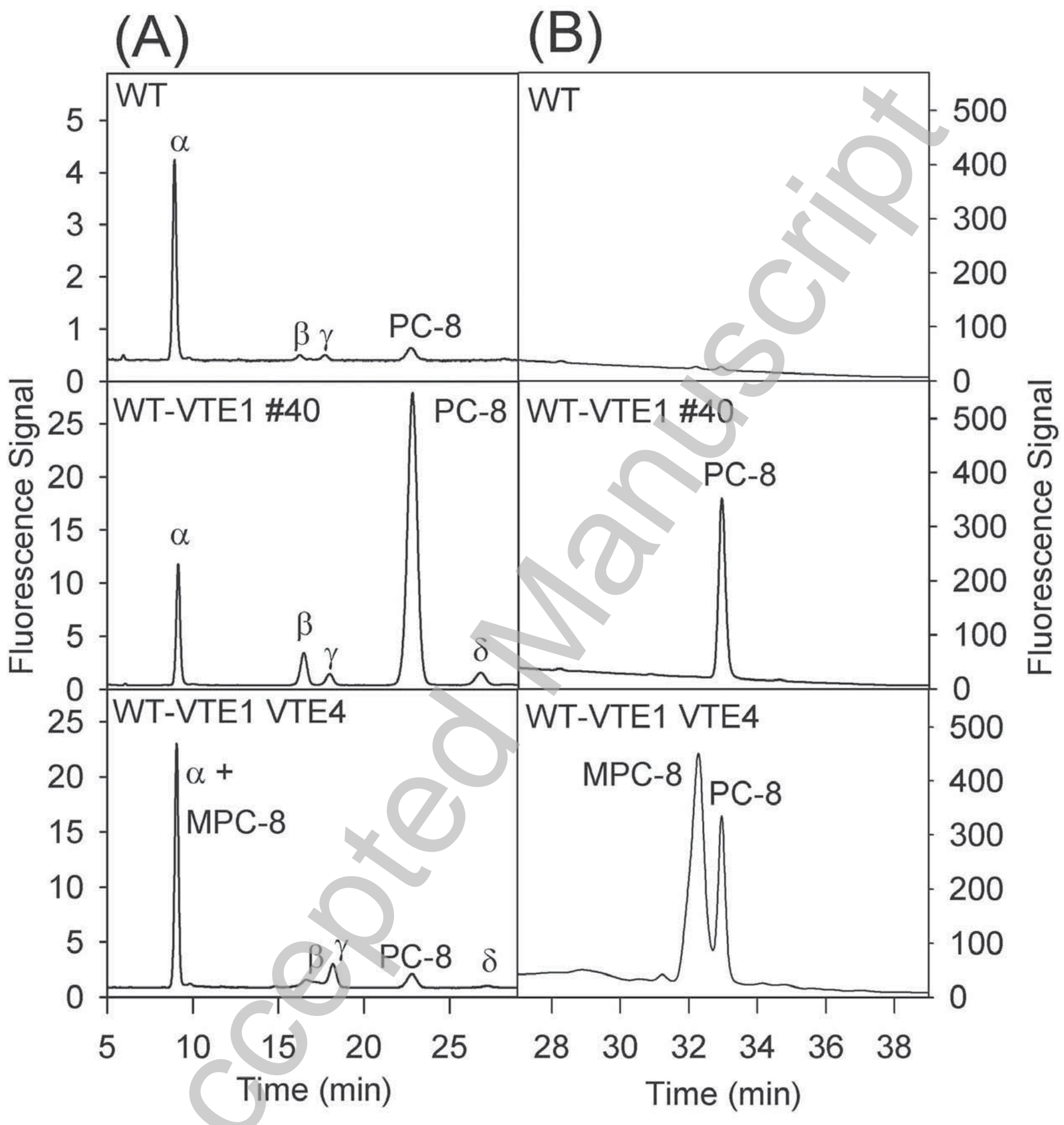

Zbierzak et al. (2009)

Fig. 2 


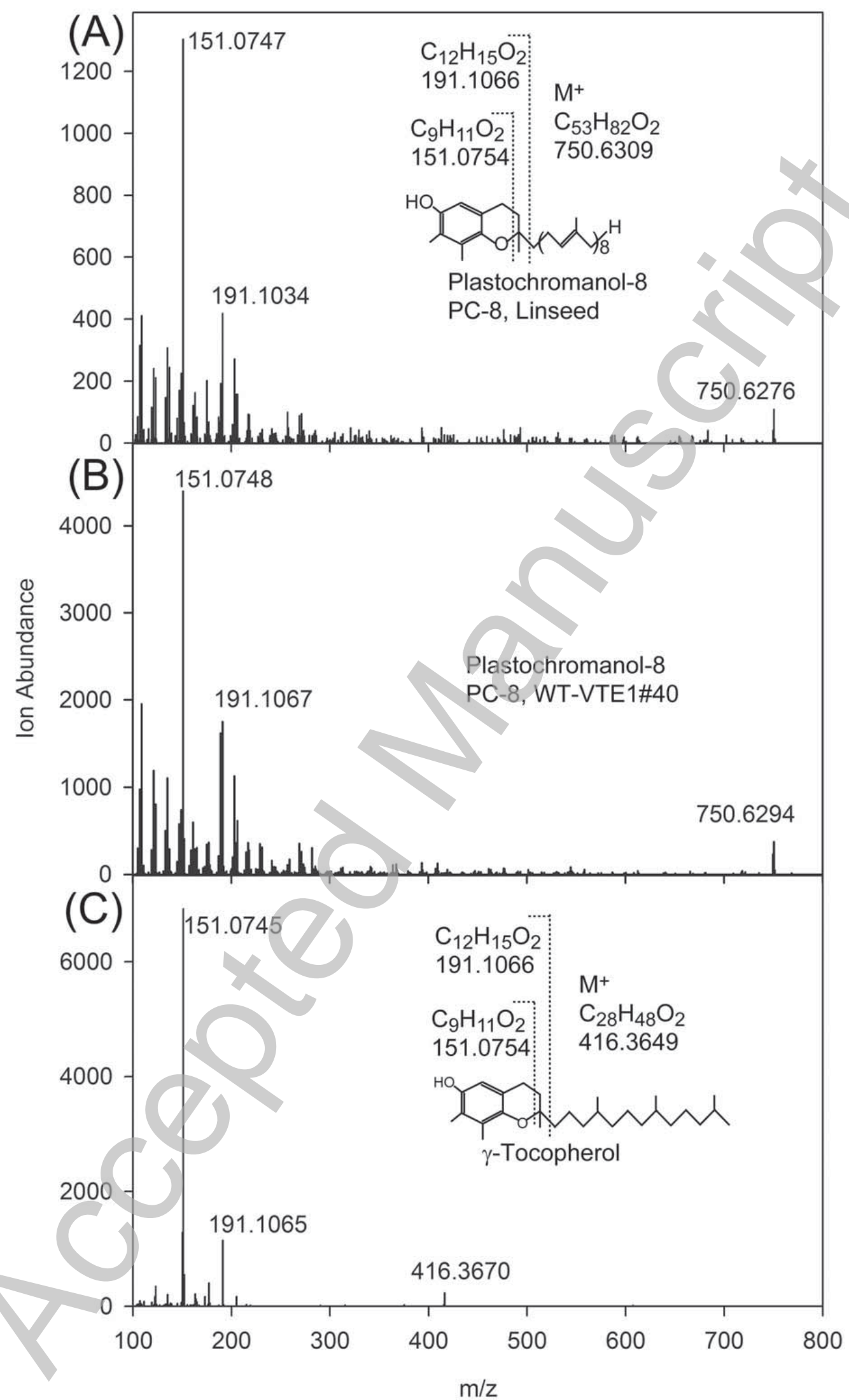

Zbierzak et al. (2009)

Figure 3 

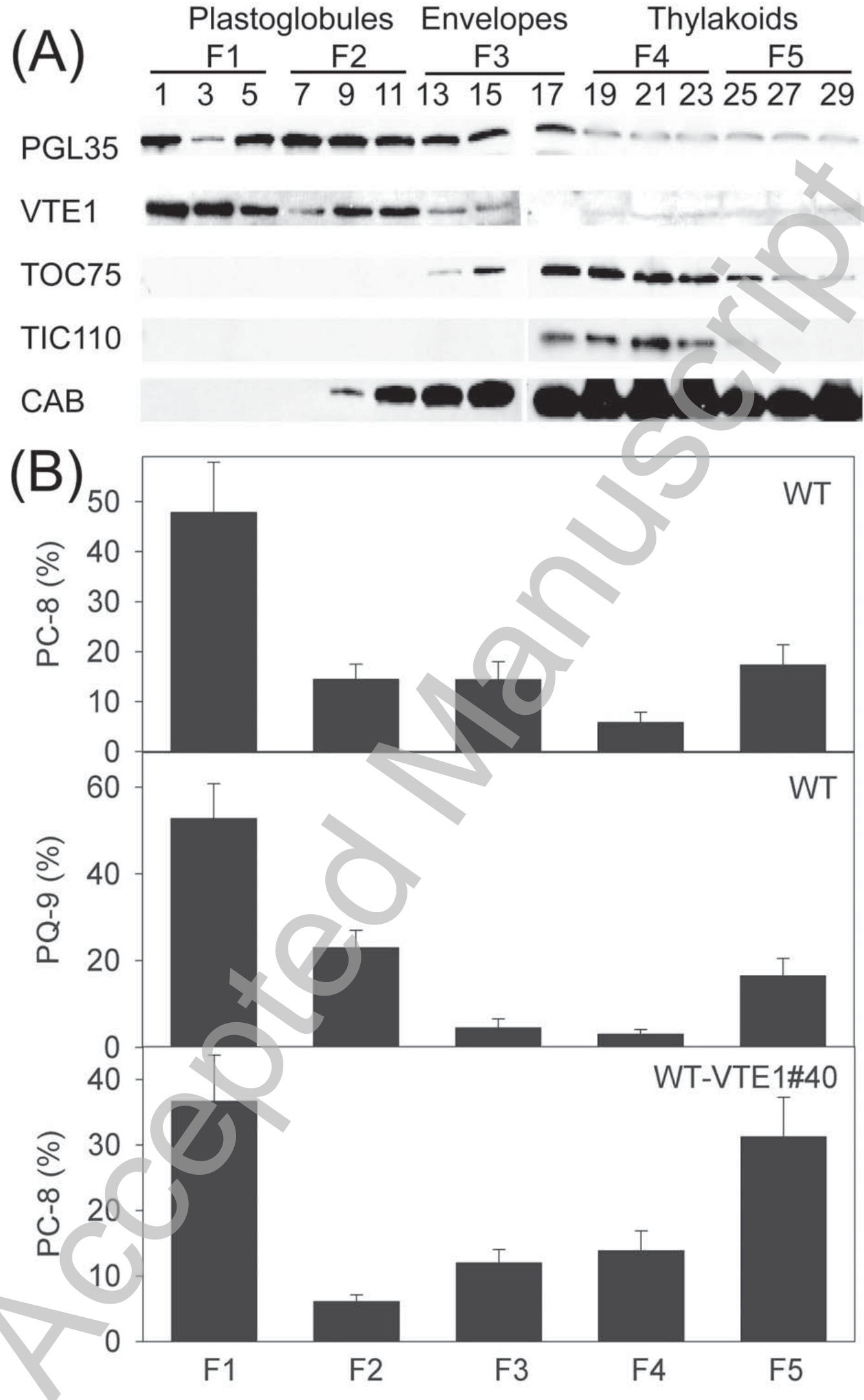

Zbierzak et al. (2009)

Figure 4 


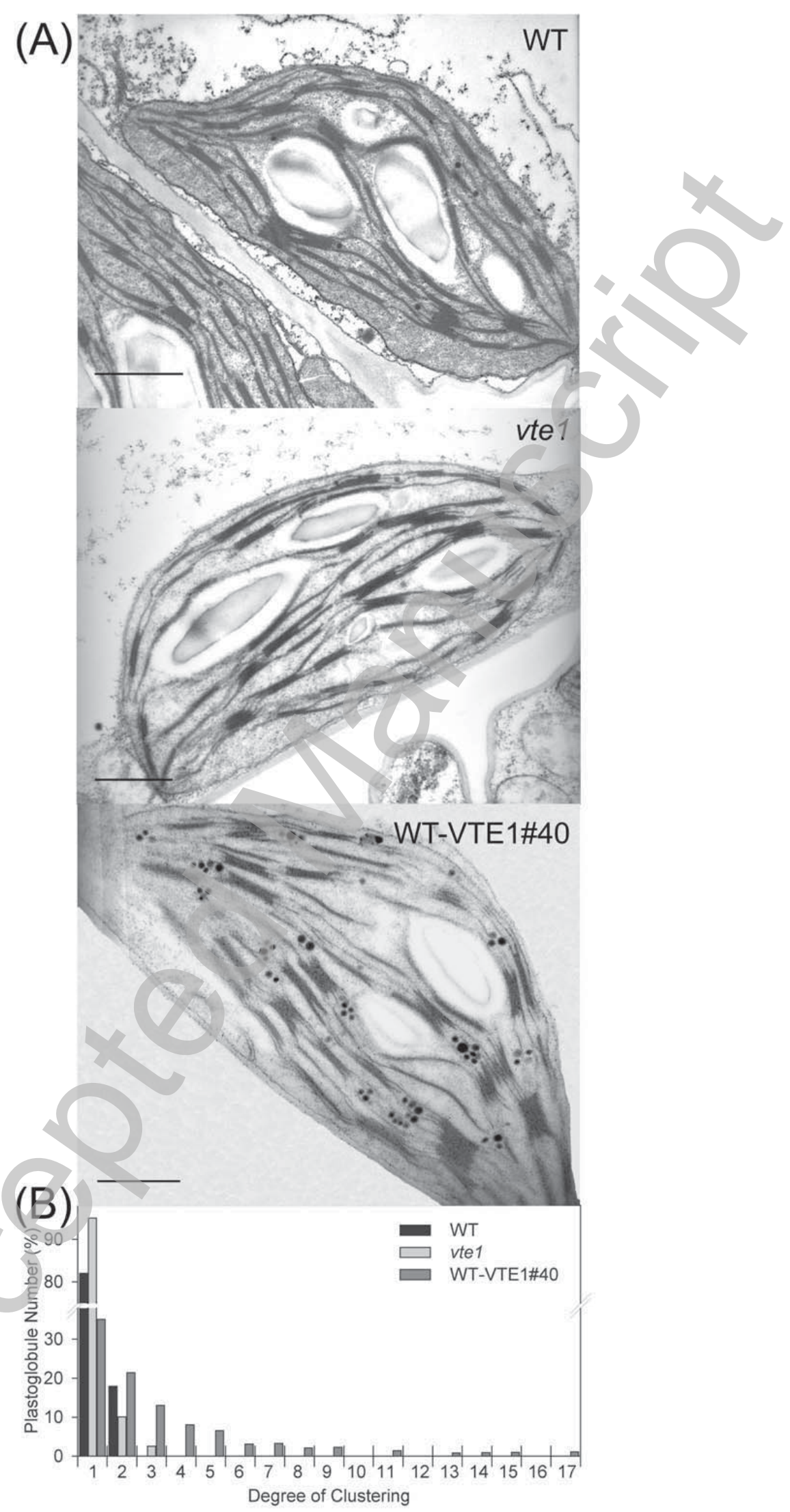

Zbierzak et al. (2009) Figure 5 


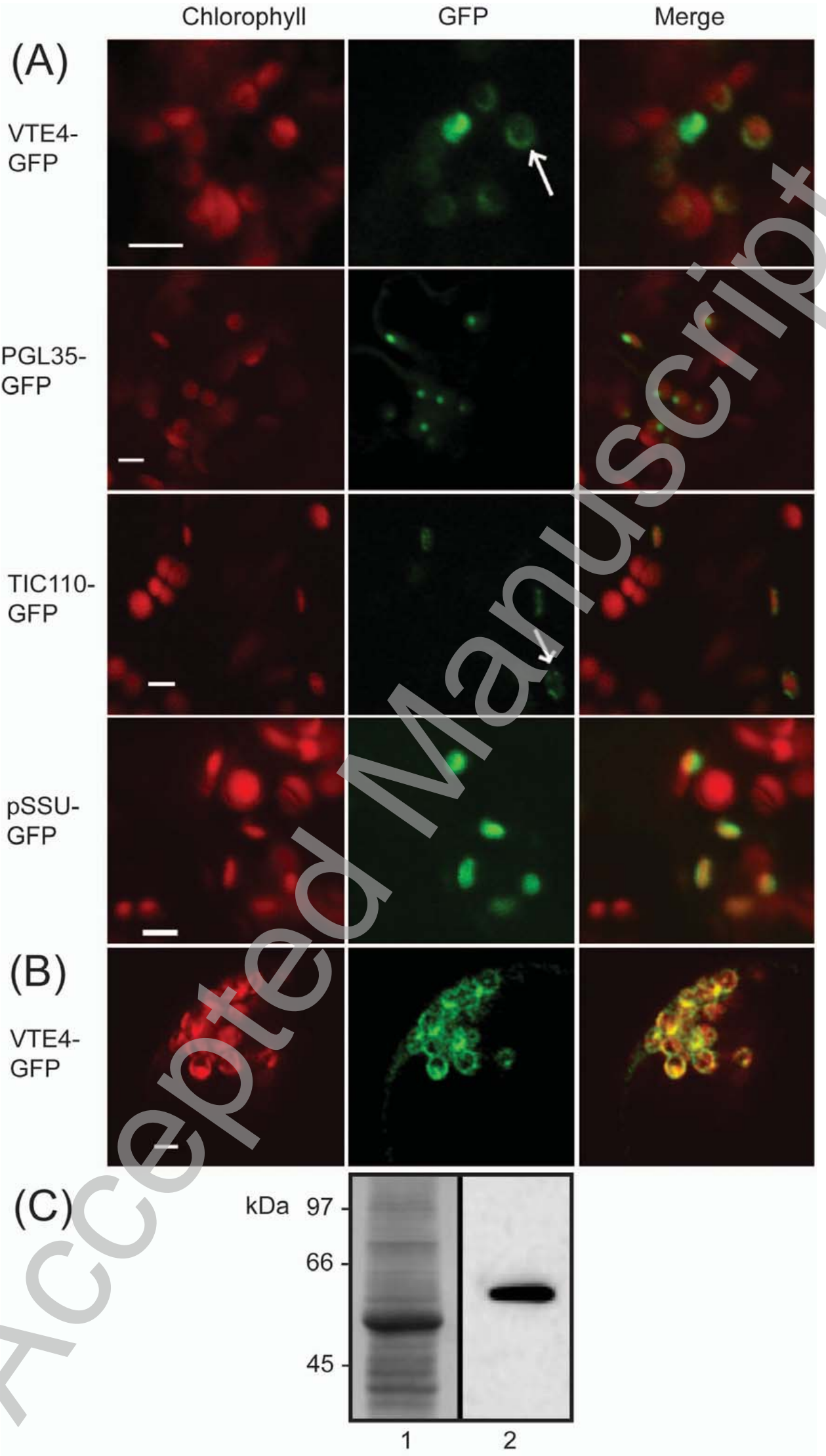

Zbierzak et al. (2009)

Fig. 6 